Pacific Journal of Mathematics

ON THE LATTICE OF NORMAL SUBGROUPS OF A DIRECT 


\title{
ON THE LATTICE OF NORMAL SUBGROUPS OF A DIRECT PRODUCT
}

\author{
Michael D. Miller
}

\begin{abstract}
Suzuki has determined that if $G$ is a direct product $G=\prod_{t=1}^{k} G_{i}$ of groups $G_{i} \neq 1$, then the lattice $L(G)$ of subgroups of $G$ is the direct product of the lattices $L\left(G_{i}\right)$ if and only if the order of any element in $G_{i}$ is finite and relatively prime to the order of any element in $G_{i}(i \neq j)$. An exercise in Zassenhaus' The Theory of Groups asks the reader to prove an analogous result for the lattice of normal subgroups. In \$1, we derive this result for the case of the direct product of two groups. (The generalization to the direct product of any finite number of groups is straightforward.) In $\$ 2$, we use results obtained in $\$ 1$ to study in detail the normal subgroup lattice of the direct product of finitely many symmetric groups.
\end{abstract}

1. The lattice of normal subgroups. If $G_{1}$ and $G_{2}$ are groups, we denote elements of the direct product $G_{1} \times G_{2}$ by ordered pairs $(a, b), a \in G_{1}, b \in G_{2}$. If $A$ and $B$ are subgroups of a group $G$, we define $[A, B]=\left\langle a b a^{-1} b^{-1} \mid a \in A, b \in B\right\rangle$, and note that if $A \triangleleft G$, then $[A, B] \triangleleft A$. We let $\rho_{1}$ and $\rho_{2}$ denote the first and second projection maps on $G_{1} \times G_{2}$, and finally, we denote by $o(g)$ the order of the element g.

If $N$ is a subgroup of $G_{1} \times G_{2}$, we put $N_{1}=\rho_{1}(N)$ and $N_{2}=$ $\rho_{2}(N)$. Thus $N_{i}$ is a subgroup of $G_{i}$, called the $i$ th projection of $N$. Furthermore, if $N \triangleleft G_{1} \times G_{2}$, then $N_{i} \triangleleft G_{i}$.

LEMMA 1. If $N \triangleleft G_{1} \times G_{2}$, then $N \supseteq\left[G_{1}, N_{1}\right] \times\left[G_{2}, N_{2}\right]$.

Proof. Let $a \in N_{1}$. Then there exists $y \in N_{2}$ such that $(a, y) \in$ $N$. Thus $\left(a^{-1}, y^{-1}\right) \in N$, and since $N \triangleleft G_{1} \times G_{2},(g, 1)(a, y)\left(g^{-1}, 1\right)=$ $\left(\mathrm{gag}^{-1}, y\right) \in N$. It follows that $\left(\mathrm{gag}^{-1}, y\right)\left(a^{-1}, y^{-1}\right)=\left(\mathrm{gag}^{-1} a^{-1}, 1\right) \in N$, so $N \supseteq\left[G_{1}, N_{1}\right] \times\{1\}$. Similarly, $N \supseteq\{1\} \times\left[G_{2}, N_{2}\right]$, completing the proof.

The following lemma, whose proof is immediate, will be used in the discussion that follows.

LemMA 2. Let $G$ be a group, $H \triangleleft G$. Then any subgroup $L$ of $G$ such that $[G, H] \subseteq L \subseteq H$ is normal in $G$.

Since $\left[G_{1} \times G_{2}, A \times B\right]=\left[G_{1}, A\right] \times\left[G_{2}, B\right]$ whenever $A \subseteq G_{1}, B \subseteq$ $G_{2}$, if $N \triangleleft G_{1} \times G_{2}$ with projections $N_{1}$ and $N_{2}$, then any subgroup of $G_{1} \times G_{2}$ lying between $\left[G_{1}, N_{1}\right] \times\left[G_{2}, N_{2}\right]$ and $N_{1} \times N_{2}$ is normal in 
$G_{1} \times G_{2}$. Moreover, as $\left[N_{i}, N_{i}\right] \subseteq\left[G_{i}, N_{i}\right]$, we see that $C_{i}=N_{i} /\left[G_{i}, N_{i}\right]$ is abelian, as is $C_{1} \times C_{2}=\left(N_{1} \times N_{2}\right) /\left[G_{1}, N_{1}\right] \times\left[G_{2}, N_{2}\right]$. There is thus a 1-1 correspondence $\phi$ between subgroups of $C_{1} \times C_{2}$ and subgroups of $G_{1} \times G_{2}$ lying between $\left[G_{1}, N_{1}\right] \times\left[G_{2}, N_{2}\right]$ and $N_{1} \times N_{2}$.

Definition. A normal subgroup $S$ of $G_{1} \times G_{2}$ is called $G_{1}-G_{2}$ decomposable if $S=S_{1} \times S_{2}, S_{1} \triangleleft G_{1}, S_{2} \triangleleft G_{2}$.

It is easy to see that a subgroup $H$ of $C_{1} \times C_{2}$ is $C_{1}-C_{2}$ decomposable if and only if $\phi(H)$ is $G_{1}-G_{2}$ decomposable. Furthermore, if $B \subseteq C_{1} \times C_{2}$, then the $i$ th projection of $B$ is $C_{i}$ if and only if the $i$ th projection of $\phi(B)$ is $N_{i}$.

Assuming we can determine the subgroup lattice structure of arbitrary abelian groups, we now have a systematic way of describing the normal subgroups of $G_{1} \times G_{2}$ in terms of those of $G_{1}$ and $G_{2}$. Namely, choose $S_{1} \triangleleft G_{1}, S_{2} \triangleleft G_{2}$ and consider the subgroups $M$ of the abelian group $S_{1} /\left[G_{1}, S_{1}\right] \times S_{2} /\left[G_{2}, S_{2}\right]$ with the property that $M_{i}=$ $S_{l} /\left[G_{i}, S_{l}\right]$. To each such $M$, there corresponds a normal subgroup $N$ of $G_{1} \times G_{2}$ with $\left[G_{1}, S_{1}\right] \times\left[G_{2}, S_{2}\right] \subseteq N \subseteq S_{1} \times S_{2}$ and $N_{i}=S_{i}$. As $S_{1}$ and $S_{2}$ run through the normal subgroups of $G_{1}$ and $G_{2}$ respectively, we obtain each normal subgroup of $G_{1} \times G_{2}$ exactly once.

It is, of course, not always easy to determine all subgroups of a given abelian group. For finite groups, however, Suzuki's result shows that it suffices to consider the case of abelian $p$-groups.

EXAMPLE. Let $G_{1}=S_{3}$ (symmetric group on 3 letters) $G_{2}=\mathbf{Z}_{2}$ (cyclic group of order 2)

We calculate the following:

$\begin{array}{llcclll}S_{1} & S_{2} & {\left[G_{1}, S_{1}\right]} & {\left[G_{2}, S_{2}\right]} & C_{1} & C_{2} & \nu \\ \mathbf{1} & \mathbf{1} & \mathbf{1} & \mathbf{1} & \mathbf{1} & \mathbf{1} & 1 \\ \mathbf{1} & \mathbf{Z}_{2} & \mathbf{1} & \mathbf{1} & \mathbf{1} & \mathbf{Z}_{2} & 1 \\ \mathbf{Z}_{3} & \mathbf{1} & \mathbf{Z}_{3} & \mathbf{1} & \mathbf{1} & \mathbf{1} & 1 \\ \mathbf{Z}_{3} & \mathbf{Z}_{2} & \mathbf{Z}_{3} & \mathbf{1} & \mathbf{1} & \mathbf{Z}_{2} & 1 \\ \mathrm{~S}_{3} & \mathbf{1} & \mathbf{Z}_{3} & \mathbf{1} & \mathbf{Z}_{2} & \mathbf{1} & 1 \\ \mathrm{~S}_{3} & \mathbf{Z}_{2} & \mathbf{Z}_{3} & \mathbf{1} & \mathbf{Z}_{2} & \mathbf{Z}_{2} & 2\end{array}$

Here $C_{i}=S_{i} /\left[G_{i}, S_{i}\right]$, and $\nu$ denotes the number of subgroups $M \subseteq$ $C_{1} \times C_{2}$ with $M_{\imath}=C_{i}$.

From this, we see that $S_{3} \times Z_{2}$ has seven normal subgroups, all of which are $S_{3}-Z_{2}$ decomposable, except for one of order six. 
We now determine the condition for every normal subgroup of $G_{1} \times G_{2}$ to be $G_{1}-G_{2}$ decomposable. Recall that a group $G$ is called perfect if $G=G^{\prime}$. We will say that $G$ is super-perfect if $[G, H]=H$ for all $H \triangleleft G$.

THEOREM 1. Let $G_{1}$ and $G_{2}$ be groups. Then every normal subgroup of $G_{1} \times G_{2}$ is $G_{1}-G_{2}$ decomposable if and only if either (i) at least one of $G_{1}$ and $G_{2}$ is super-perfect, or (ii) for all $S_{1} \triangleleft G_{1}, S_{2} \triangleleft G_{2}$, the elements of $S_{1} /\left[G_{1}, S_{1}\right]$ have orders relatively prime to those of $S_{2} /\left[G_{2}, S_{2}\right]$. (In particular, these orders must be finite.)

Proof. $(\Leftarrow)$ Suppose $N \triangleleft G_{1} \times G_{2}$ is not $G_{1}-G_{2}$ decomposable. Then the subgroup $\phi(N)$ of $N_{1} /\left[G_{1}, N_{1}\right] \times N_{2} /\left[G_{2}, N_{2}\right]=C_{1} \times C_{2}$ is not $C_{1}-C_{2}$ decomposable. If $G_{i}$ is super-perfect, then $C_{i}=1$, a contradiction. Otherwise (ii) holds, and we have a contradiction to Suzuki's result [2]. $(\Rightarrow)$ Let $S_{i} \triangleleft G_{i}$. By hypothesis, every normal subgroup $N \subseteq G_{1} \times G_{2}$ with $\left[G_{1}, S_{1}\right] \times\left[G_{2}, S_{2}\right] \subseteq N \subseteq S_{1} \times S_{2}$ is $G_{1}-G_{2}$ decomposable, and therefore all subgroups of $S_{1} /\left[G_{1}, S_{1}\right] \times S_{2} /\left[G_{2}, S_{2}\right]=$ $C_{1} \times C_{2}$ are $C_{1}-C_{2}$ decomposable. If $C_{1}$ has elements of infinite order, then $G_{2}$ must be super-perfect. For if not, there is a normal subgroup $H$ of $G_{2}$ such that $D=H /\left[G_{2}, H\right] \neq 1$. By Suzuki's result, $C_{1} \times D$ contains a subgroup which is not $C_{1}-D$ decomposable, a contradiction. Simililarly, if $G_{1}$ is not super-perfect, then $C_{2}$ must be a torsion group.

Finally, if neither $G_{1}$ nor $G_{2}$ is super-perfect, then the order of any element in $C_{1}$ must be relatively prime to the order of any element in $C_{2}$, for if not, by Suzuki's result, there would be a subgroup of $C_{1} \times C_{2}$ which is not $C_{1}-C_{2}$ decomposable.

CoROllaRY 1. Every normal subgroup of $G \times G$ is $G-G$ decomposable if and only if $G$ is super-perfect.

COROLlARY 2. If $G_{1}$ and $G_{2}$ are torsion groups, and the order of any element in $G_{1}$ is relatively prime to the order of any element in $G_{2}$, then every normal subgroup of $G_{1} \times G_{2}$ is $G_{1}-G_{2}$ decomposable.

Definition. A torsion group $G$ is called quasi-nilpotent if for every prime $p$ with $p=o(a)$ for some $a \in G$, there exists $H \triangleleft G$ such that $H /[G, H]$ has an element of order $p$.

It is easy to see that every nilpotent torsion group is quasinilpotent. The first example of a quasi-nilpotent group which is not nilpotent is the group $S_{3} \times \mathbf{Z}_{3}$, of order 18 . The first indecomposable example is $\operatorname{SL}(2,3)$ of order 24. 
We now state a partial converse to Corollary 2:

COROLlaRy 3. If $G_{1}$ and $G_{2}$ are quasi-nilpotent groups such that every normal subgroup of $G_{1} \times G_{2}$ is $G_{1}-G_{2}$ decomposable, then the order of any element in $G_{1}$ is relatively prime to the order of any element in $G_{2}$.

Theorem 1 can be easily generalized to yield the following:

THEOREM. 2. Let $G=\prod_{i=1}^{k} G_{i}$. Then every normal subgroup $N$ of $G$ is a direct product $N=\prod_{i=1}^{k} N_{i}$ of normal subgroups $N_{i}$ of $G_{i}$ if and only if either (i) at most one of the $G_{i}$ is not super-perfect, or (ii) whenever $H_{i} \triangleleft G_{i}$ and $H_{j} \triangleleft G_{j}(i \neq j)$, the order of any element in $H_{i} /\left[G_{i}, H_{i}\right]$ is relatively prime to the order of any element in $H_{j} /\left[G_{j}, H_{j}\right]$. (In particular, these orders must be finite.)

The proof is identical in nature to that of Theorem 1, and will therefore be omitted.

Instead of studying the lattice of normal subgroups, one can look at other systems of subgroups which form a lattice. For example, one could ask when every characteristic (resp. fully invariant) subgroup of $\Pi_{i=1}^{k} G_{i}$ is a direct product of characteristic (resp. fully invariant) subgroups of the individual $G_{i}$. These problems appear to be substantially more difficult than the one treated in this section.

2. Direct products of symmetric groups. We begin with two definitions. If $G$ is a group and $H$ is any subgroup containing $G^{\prime}$, then $H$ is called a $C C$-subgroup of $G$. All $C C$-subgroups are therefore normal. Secondly, if $G=\prod_{i=1}^{k} G_{i}$ and $\rho_{i}$ is the projection on the $i$ th factor, then an automorphism $\phi$ of $G$ is called rigid if $\phi\left(\rho_{i}(G)\right)=$ $\rho_{i}(G)$ for all $i$. The group of rigid automorphisms of $G$ is thus isomorphic to $\prod_{i=1}^{k} \operatorname{Aut}\left(G_{i}\right)$.

Now let $\left(S_{n}\right)^{k}$ be the direct product of $k$ copies of the symmetric group $S_{n}$, where $n>4$. (The results of this section are not in general true for $n \leqq 4$, although analogous results may be obtained by treating each case separately.)

We wish to determine all normal subgroups of $\left(S_{n}\right)^{k}$. For $k=1$, there are exactly three: $1, A_{n}$, and $S_{n}$. Suppose the normal subgroups of $\left(S_{n}\right)^{r}$ for $r<k$ have been determined. Then if $N \triangleleft\left(S_{n}\right)^{k}$, we may assume that $N$ is not contained in a product of fewer than $k$ copies of $S_{n}$. By the simplicity of $A_{n}, N \supseteq\left(A_{n}\right)^{k}$ and so $N$ is a $C C$-subgroup.

Now $\left(S_{n}\right)^{k} /\left(A_{n}\right)^{k}$ is an elementary abelian 2-group, which may be considered as the vector space $P$ (over $Z_{2}$ ) of subsets of the set $K=\{1,2, \cdots, k\}$, where addition is defined by symmetric difference. There is therefore a $1-1$ correspondence $\sigma$ between $C C$ - 
subgroups of $\left(S_{n}\right)^{k}$ and subspaces of $P$. We proceed to show that this correspondence can be made canonical.

If $N$ is a $C C$-subgroup of $\left(S_{n}\right)^{k}$, let $\sigma(N)$ be the subspace of $P$ spanned by the set of all $U \subseteq K$ such that $\Pi_{i \in U} x_{i} \in A_{n}$ for all $\left(x_{1}, \cdots, x_{k}\right) \in N$. Conversely, if $S$ is a subspace of $P$, let $N$ be that $C C$ subgroup of $\left(S_{n}\right)^{k}$ consisting of all elements $\left(x_{1}, x_{2}, \cdots, x_{k}\right)$ such that $\prod_{i \in R} x_{i} \in A_{n}$ for all $R \in S$. It is easily verified that $\sigma(N)=S$, and that $\sigma$ is a Galois correspondence, i.e., it is $1-1$ and reverses inclusion.

For example, $\left(S_{n}\right)^{2} /\left(A_{n}\right)^{2}$ is isomorphic to the Klein group $\mathbf{Z}_{2} \times \mathbf{Z}_{2}$, which has 5 subgroups (subspaces). There are therefore $5 \mathrm{CC}$ subgroups of $\left(S_{n}\right)^{2}$ viz., $\left(A_{n}\right)^{2}, A_{n} \times S_{n}, S_{n} \times A_{n},\left\{\left(x_{1}, x_{2}\right) \mid x_{1} x_{2} \in A_{n}\right\}$, and $\left(S_{n}\right)^{2}$. If we add to these the subgroups $1 \times 1,1 \times A_{n}, A_{n} \times 1,1 \times S_{n}$, and $S_{n} \times 1$, we find that there is a total of 10 normal subgroups in $\left(S_{n}\right)^{2}$.

Now $e_{1}, e_{2}, \cdots, e_{k}$ where $e_{i}=\{i\}$ form a basis of $P$. Under $\sigma, e_{i}$ corresponds to the subgroup of all $\left(x_{1}, x_{2}, \cdots, x_{k}\right) \in\left(S_{n}\right)^{k}$ such that $x_{i}$ is even. We define a coordinate plane to be a subspace of $P$ spanned by some collection of the $e_{i}$, and call it proper if it has dimension $<k$. It is not hard to see that a $C C$-subgroup $N$ of $\left(S_{n}\right)^{k}$ is a nontrivial direct product of two normal subgroups of $\left(S_{n}\right)^{k}$ if and only if $\sigma(N)$ is the direct sum of two subspaces of $P$, contained respectively in complementary proper coordinate planes.

We now recall the following well-known facts:

(i) For $n>4, n \neq 6$, Aut $S_{n} \cong$ Aut $A_{n} \cong S_{n}$. Moreover, Aut $S_{6} \cong$ Aut $A_{6}$ and [Aut $S_{6}$ : Inn $S_{6}$ ] $=2$.

(ii) (Mathewson [1]) For $n>4, \quad \operatorname{Aut}\left(S_{n}\right)^{k} \cong \operatorname{Aut}\left(A_{n}\right)^{k} \cong$ (Aut $\left.S_{n}\right)^{k} x_{s} S_{k}$.

In words, every automorphism of $S_{n}(n>4, n \neq 6)$ is inner, while every automorphism of $\left(S_{n}\right)^{k}(n>4, n \neq 6)$ is the product of an inner automorphism and an automorphism which permutes the $k$ factors. For $n>4$, the automorphism group of $A_{n}$ (resp. $\left.\left(A_{n}\right)^{k}\right)$ is the same as that of $S_{n}$ (resp. $\left.\left(S_{n}\right)^{k}\right)$.

THEOREM 3. Let $N$ be a CC-subgroup of $\left(S_{n}\right)^{k}$. Then every automorphism of $N$ is induced by an automorphism of $\left(S_{n}\right)^{k}$.

Proof. Let $\theta \in$ Aut $N$. By the above, the action of $\theta$ on $\left(A_{n}\right)^{k}$ is that of the product of a rigid automorphism of $\left(A_{n}\right)^{k}$ and an automorphism which permutes the $k$ factors of $\left(A_{n}\right)^{k}$. Multiplying $\theta$ by a rigid automorphism of $\left(S_{n}\right)^{k}$ (itself an automorphism of $N$ ), we may assume that the action of $\theta$ on $\left(A_{n}\right)^{k}$ is simply a permutation $\pi$ of the $k$ factors.

Let $x \in N$ with $\theta(x)=y$ and $e \in\left(A_{n}\right)^{k}$ with $\theta(e)=e^{\pi}$. Since $x^{-1} e x \in\left(A_{n}\right)^{k}$, we have $\theta\left(x^{-1} e x\right)=x^{-\pi} e^{\pi} x^{\pi}$. But also $\theta\left(x^{-1} e x\right)=$ $y^{-1} e^{\pi} y$. As $e$ is arbitrary in $\left(A_{n}\right)^{k}, x^{\pi} y^{-1}$ is in the centralizer of $\left(A_{n}\right)^{k}$, which is trivial, so $y=x^{\pi}$. Thus every automorphism of $N$ is the 
product of a rigid automorphism of $\left(S_{n}\right)^{k}$ and an automorphism which permutes the $k$ factors. The theorem follows.

In general, not all automorphisms of $\left(S_{n}\right)^{k}$ actually restrict to automorphisms of a given $C C$-subgroup $N$, since not all permutations of the $k$ factors leave $N$ invariant. If $S=\sigma(N)$ is the corresponding subspace of $P$, let $\Gamma$ denote the group of permutation matrices (with respect to the basis $e_{1}, e_{2}, \cdots, e_{k}$ ) which leave $S$ invariant. Then Aut $N \cong\left(\text { Aut } S_{n}\right)^{k} x_{s} \Gamma$.

THEOREM 4. The characteristic subgroups of $\left(S_{n}\right)^{k}$ are:

$1,\left(S_{n}\right)^{k},\left(A_{n}\right)^{k}, T_{1}$, and $T_{2}$, where $T_{1}=\left\{\left(x_{1}, x_{2}, \cdots, x_{k}\right) \mid \Pi_{i=1}^{k} x_{i} \in A_{n}\right\}$, and $T_{2}=\left\{\left(x_{1}, x_{2}, \cdots, x_{k}\right) \mid \Pi_{i<j} x_{i} x_{j} \in A_{n}\right.$ for all $\left.i, j\right\}$. (Note that $T_{1}=T_{2}$ in case $k=2$.)

Proof. It is clear that except for 1, any characteristic subgroup of $\left(S_{n}\right)^{k}$ contains $\left(A_{n}\right)^{k}$, for otherwise it would be contained in a direct product of fewer than $k$ copies of $S_{n}$, and hence not be characteristic. In terms of $P$, we must show that the only subspaces $S$ invariant under all permutations of the coordinates are $\varnothing, P$, the 1-dimensional subspace $V_{1}$ spanned by $e_{1}+e_{2}+\cdots+e_{k}$, and the $(k-1)$-dimensional subspace $V_{2}$ spanned by all $e_{\imath}+e_{r}$.

That these are all invariant is immediate. Assume now that $S$ is invariant, and suppose that $a_{1} e_{1}+\cdots+a_{r} e_{r}+\cdots+a_{s} e_{s}+\cdots+a_{k} e_{k} \in S$, where $a_{r}+a_{s} \neq 0$ for some choice of $r$ and $s$ (otherwise $S=\varnothing$ or $\left.S=V_{1}\right)$. By invariance, $a_{1} e_{1}+\cdots+a_{r} e_{s}+\cdots+a_{s} e_{r}+\cdots+a_{k} e_{k} \in S$, and adding gives $\left(a_{r}+a_{s}\right) e_{r}+\left(a_{r}+a_{s}\right) e_{s} \in S$, so that $e_{r}+e_{s} \in S$. Again by invariance, we conclude that $e_{i}+e_{j} \in S$ for all $i, j$, so either $S=P$ or $S=V_{2}$.

The question of determining exactly which groups can arise as the group $\Gamma$ of "admissible" permutation matrices for a given $C C$-subgroup $N$ will be dealt with in a future paper.

\section{REFERENCES}

1. L. C. Mathewson, Theorems on the groups of isomorphisms of certain groups, Amer. J. Math., 38 (1916), 19-44.

2. M. Suzuki, Structure of a Group and the Structure of its Lattice of Subgroups, Springer-Verlag, 1967.

3. H. Zassenhaus, The Theory of Groups, Chelsea, 1958.

Received September 3, 1974, and in revised form October 14, 1974.

UNIVERSITY of CALIForNiA, BERKELEY 


\section{PACIFIC JOURNAL OF MATHEMATICS}

\section{EDITORS}

RICHARD ARENS (Managing Editor)

University of California

Los Angeles, California 90024

\author{
R. A. Beaumont \\ University of Washington \\ Seattle, Washington 98105
}

\section{J. DugundII}

Department of Mathematics

University of Southern California

Los Angeles, California 90007

D. Gilbarg and J. Milgram

Stanford University

Stanford, California 94305

\section{ASSOCIATE EDITORS}
E. F. BECKENBACH
B. H. NeumanN
F. WoLF
K. YoshidA

\section{SUPPORTING INSTITUTIONS}

\author{
UNIVERSITY OF BRITISH COLUMBIA \\ CALIFORNIA INSTITUTE OF TECHNOLOGY \\ UNIVERSITY OF CALIFORNIA \\ MONTANA STATE UNIVERSITY \\ UNIVERSITY OF NEVADA \\ NEW MEXICO STATE UNIVERSITY \\ OREGON STATE UNIVERSITY \\ UNIVERSITY OF OREGON \\ OSAKA UNIVERSITY
}

\author{
UNIVERSITY OF SOUTHERN CALIFORNIA \\ STANFORD UNIVERSITY \\ UNIVERSITY OF TOKYO \\ UNIVERSITY OF UTAH \\ WASHINGTON STATE UNIVERSITY \\ UNIVERSITY OF WASHINGTON \\ AMERICAN MATHEMATICAL SOCIETY
}

The Supporting Institutions listed above contribute to the cost of publication of this Journal, but they are not owners or publishers and have no responsibility for its contents or policies.

Mathematical papers intended for publication in the Pacific Journal of Mathematics should be in typed form or offset-reproduced (not dittoed), double spaced with large margins. Underline Greek letters in red, German in green, and script in blue. The first paragraph or two must be capable of being used separately as a synopsis of the entire paper. Items of the bibliography should not be cited there unless absolutely necessary, in which case they must be identified by author and Journal, rather than by item number. Manuscripts, in duplicate, may be sent to any one of the four editors. Please classify according to the scheme of Math. Reviews, Index to Vol. 39. All other communications should be addressed to the managing editor, or Elaine Barth, University of California, Los Angeles, California, 90024.

100 reprints are provided free for each article, only if page charges have been substantially paid. Additional copies may be obtained at cost in multiples of 50.

The Pacific Journal of Mathematics is issued monthly as of January 1966. Regular subscription rate: $\$ 72.00$ a year (6 Vols., 12 issues). Special rate: $\$ 36.00$ a year to individual members of supporting institutions.

Subscriptions, orders for back numbers, and changes of address should be sent to Pacific Journal of Mathematics, 103 Highland Boulevard, Berkeley, California, 94708.

PUBLISHED BY PACIFIC JOURNAL OF MATHEMATICS, A NON-PROFIT CORPORATION Printed at Jerusalem Academic Press, POB 2390, Jerusalem, Israel.

\section{Copyright (C) 1975 Pacific Journal of Mathematics All Rights Reserved}




\section{Pacific Journal of Mathematics

Vol. 60, No. $2 \quad$ October, 1975

Waleed A. Al-Salam and A. Verma, A fractional Leibniz q-formula ........... 1

Robert A. Bekes, Algebraically irreducible representations of $L_{1}(G) \ldots \ldots \ldots \ldots 11$

Thomas Theodore Bowman, Construction functors for topological

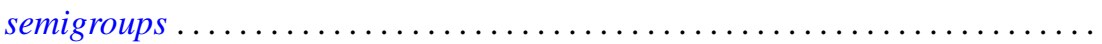

Stephen LaVern Campbell, Operator-valued inner functions analytic on the

closed disc. II .........................................

Leonard Eliezer Dor and Edward Wilfred Odell, Jr., Monotone bases in $L_{p} \ldots \ldots$.

Yukiyoshi Ebihara, Mitsuhiro Nakao and Tokumori Nanbu, On the existence of

global classical solution of initial-boundary value problem for

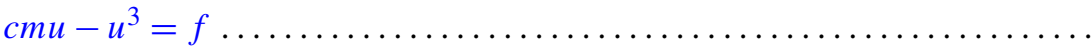

Y. Gordon, Unconditional Schauder decompositions of normed ideals of

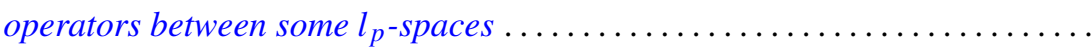

Gary Grefsrud, Oscillatory properties of solutions of certain nth order functional

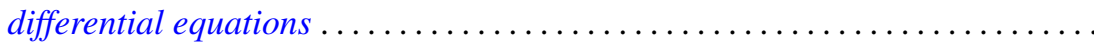

Irvin Roy Hentzel, Generalized right alternative rings ...................

Zensiro Goseki and Thomas Benny Rushing, Embeddings of shape classes of compacta in the trivial range .................................

Emil Grosswald, Brownian motion and sets of multiplicity . .

Donald LaTorre, A construction of the idempotent-separating congruences on a

bisimple orthodox semigroup .

Pjek-Hwee Lee, On subrings of rings with involution ...

Marvin David Marcus and H. Minc, On two theorems of Frobenius ...

Michael Douglas Miller, On the lattice of normal subgroups of a direct

product. .

Grattan Patrick Murphy, A metric basis characterization of Euclidean space

Roy Martin Rakestraw, A representation theorem for real convex functions ....

Louis Jackson Ratliff, Jr., On Rees localities and $H_{i}$-local rings ...

Simeon Reich, Fixed point iterations of nonexpansive mapping . .

Domenico Rosa, $B$-complete and $B_{r}$-complete topological algebras ...

Walter Roth, Uniform approximation by elements of a cone of real-valued

functions ....

Helmut R. Salzmann, Homogene kompakte projektive Ebenen

Jerrold Norman Siegel, On a space between $B H$ and $B_{\infty} \ldots$

235

Robert C. Sine, On local uniform mean convergence for Markov operators

James D. Stafney, Set approximation by lemniscates and the spectrum of an

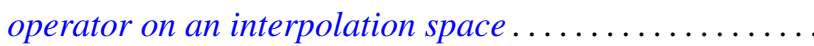

Árpád Száz, Convolution multipliers and distributions .......

Kalathoor Varadarajan, Span and stably trivial bundles ..........

Robert Breckenridge Warfield, Jr., Countably generated modules over

commutative Artinian rings....................... 